\title{
Análise qualitativa do método de pseudo decomposição de tiros blended empregando diferentes condições de imagem
}

Karen Carrilho da Silva*1 - karenkrrilho@gmail.com

André Bulcão², Luiz Landau ${ }^{3}$, Josias José da Silva1

${ }^{1}$ Lab2M/COPPE/Universidade Federal do Rio de Janeiro, ${ }^{2}$ Centro de Pesquisas da Petrobras,

${ }^{3}$ LAMCE/COPPE/Universidade Federal do Rio de Janeiro

Copyright 2012, SBGf - Sociedade Brasileira de Geofísica

Este texto foi preparado para a apresentação no V Simpósio Brasileiro de Geofísica, Salvador, 27 a 29 de novembro de 2012. Seu conteúdo foi revisado pelo Comite Técnico do V SimBGf, mas não necessariamente representa a opinião da SBGf ou de seus associados. É proibida a reprodução total ou parcial deste material para propósitos comerciais sem prévia autorização da SBGf.

\section{Abstract}

This paper presents the pseudo deblending scheme (Mahdad et al., 2011) and the results of applying Reverse Time Migration using different imaging conditions for this kind of seismic data. Moreover it compares images from blended seismic data with images from pseudo deblended seismic data in terms of quality and computational cost.

In general, the results using pseudo deblended seismic data are satisfactory because they present similar quality of those from blended seismic data. However, processing time and computational cost requirements for data storage were higher.

2D synthetic model with horizontal interfaces was used for presentation of the pseudo deblending scheme, as well as for the achieved results.

\section{Introdução}

A aquisição ou simulação de dados sísmicos blended, termo introduzido por Berkhout (2008), consiste da geração de ondas sísmicas em várias posições em intervalos de tempo menores que a duração do registro do dado sísmico. Esse intervalo de tempo pequeno entre tiros consecutivos resulta na superposição dos sinais, causando interferência no dado registrado.

Teoricamente, a interferência entre as diversas fontes sísmicas pode ser eliminada ou atenuada durante a etapa de processamento sísmico por um método chamado deblending ou decomposição de tiros blended (lkelle, 2007). Este método consiste na reconstrução do dado como se a aquisição fosse realizada de maneira convencional (ondas tendo sido geradas de uma única fonte e a resposta sísmica sendo registrada antes do próximo tiro ser disparado). Deste modo, consegue-se decompor o dado blended e transformá-lo em dados convencionais, não havendo assim a superposição de sinais, eliminando a interferência entre os tiros.
A decomposição de tiros é um método computacionalmente custoso e com grandes desafios, como por exemplo, a obtenção da assinatura da fonte sísmica de forma precisa e a resposta impulsiva do meio (Ikelle, 2007). Uma alternativa mais rápida e simples é o método pseudo deblending ou pseudo decomposição de tiros blended.

O objetivo deste trabalho é comparar as imagens obtidas de dados sísmicos blended com as obtidas de dados sísmicos pseudo de-blended em termos de qualidade e custo computacional requerido utilizando condição de imagem de tempo de excitação e condição de imagem de correlação cruzada, ambas baseadas no princípio do imageamento proposto por Claerbout (1971).

\section{Metodologia/ Problema Investigado}

Para a simulação de dados sísmicos blended foram gerados 10 sismogramas sintéticos oriundos da modelagem numérica de 10 experimentos, cada um com 10 tiros blended. Esses 10 tiros estão a uma distância de $357 \mathrm{~m}$ entre si e os 10 experimentos foram escolhidos de modo a percorrer todo o modelo de velocidades apresentado na figura 1 a seguir.

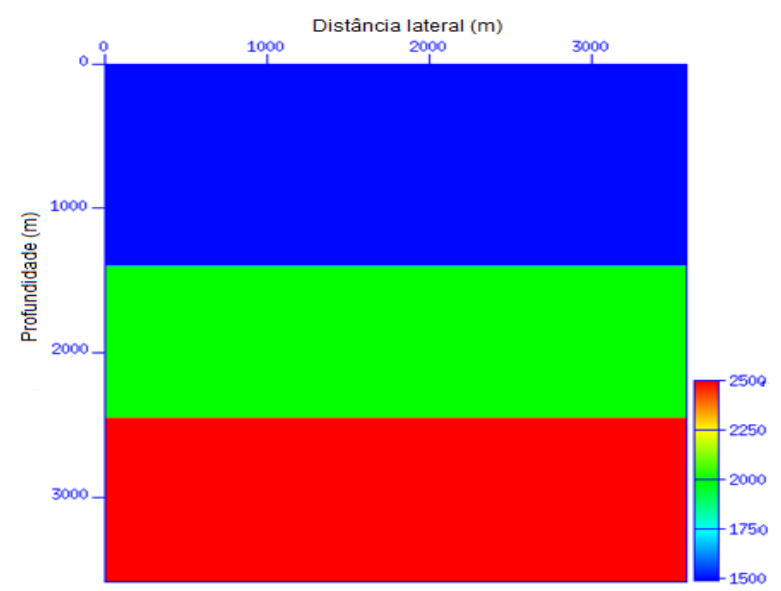

Figura 1 - Modelo de velocidades. 
O método de pseudo decomposição de tiros consiste de se escolher uma determinada posição de fonte em um sismograma blended e eliminar o atraso da mesma dando um shift no sismograma, de modo a nessa posição o registro do dado sísmico começar a partir de $\mathrm{t}=0 \mathrm{~s}$, como apresentado na figura 2 . Posteriormente, acrescentam-se zeros na parte inferior do sismograma de modo a compensar o shift dado anteriormente.

Caso o sismograma contenha o registro do sinal sísmico gerado a partir de $\mathrm{N}$ fontes, o resultado da aplicação deste método são $\mathrm{N}$ sismogramas processados. É importante observar que, neste esquema de pseudo deblending não se consegue decompor todo 0 dado blended e eliminar a interferência entre as fontes, como pode ser observado na figura 2.

Em seguida, na etapa de migração utilizam-se os $\mathrm{N}$ sismogramas pseudo de-blended como dado de entrada e migra-se cada um como se a aquisição fosse realizada de forma convencional, um sismograma de cada vez. Note que, ao migrar um sismograma pseudo de-blended apenas um sismograma contido neste funcionará como sinal e os outros funcionarão como ruído na geração da imagem em profundidade.
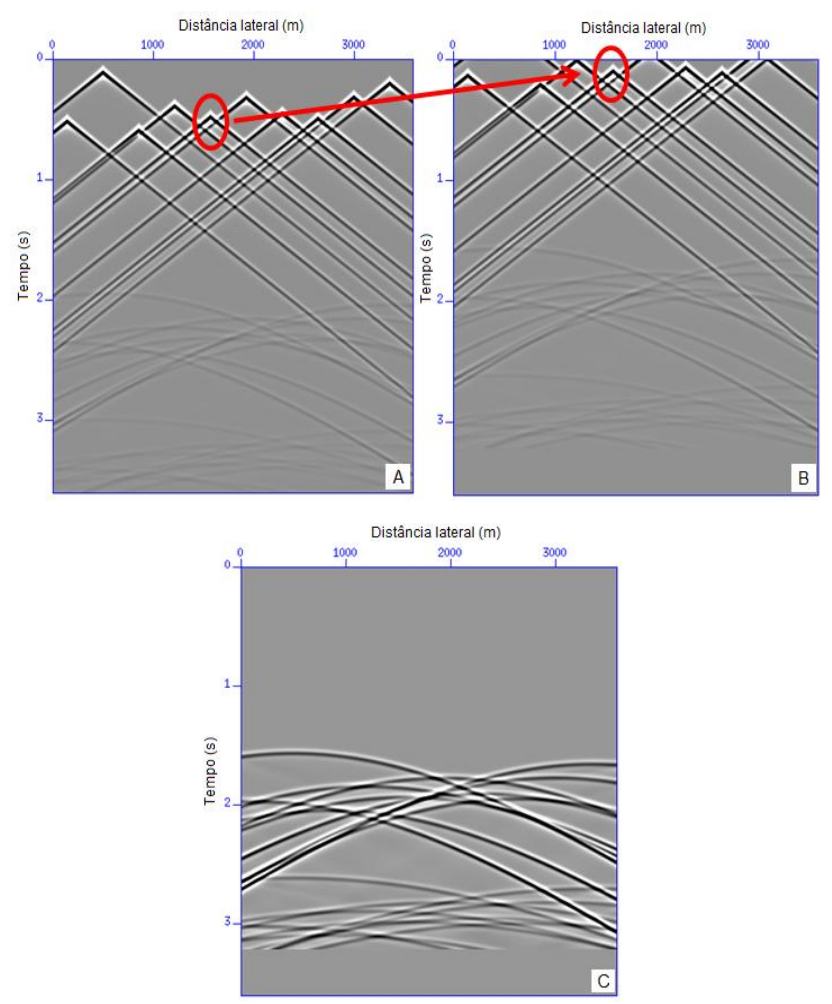

Figura 2 - Apresentação do método de pseudo decomposição de tiros: A)sismograma com 10 tiros blended; B)sismograma pseudo de-blended e C)sismograma pseudo de-blended sem a onda direta.

\section{Resultados}

Com o objetivo de analisar a qualidade das imagens em profundidade o sismograma pseudo de-blended sem a onda direta apresentado na figura anterior foi migrado utilizando o esquema de Migração Reversa no Tempo (RTM), empregando a condição de imagem de tempo de excitação utilizando o critério da amplitude máxima no cálculo da matriz tempo de trânsito e a condição de imagem de correlação cruzada. $\mathrm{Na}$ figura 3 são apresentadas as imagens resultantes.

É importante destacar que, para a implementação computacional do esquema de Migração Reversa no Tempo foi utilizada uma borda randômica em torno do domínio computacional, método proposto por Clapp (2009).

Nestas imagens pode-se observar que:

- 1 sismograma com 1 tiro pseudo de-blended consegue imagear apenas uma parte das verdadeiras interfaces, destacadas por um círculo amarelo;

- Como neste sismograma apenas o sinal referente a 1 tiro funciona como sinal e o sinal referente aos outros 9 tiros funcionam, na verdade, como ruído, este pode ser verificado em grande quantidade e de forma coerente na imagem;

- As diferentes condições de imagem não apresentam divergências em relação à quantidade de ruído coerente gerado.

Posteriormente, o método de pseudo decomposição de tiros foi aplicado em todas as 10 posições de fonte do sismograma apresentado na figura 2 originando 10 sismogramas pseudo de-blended e foi eliminada a influência da onda direta em cada sismograma antes da etapa de migração. Na figura 4 são apresentadas as imagens finais oriundas do empilhamento das 10 imagens geradas a partir da migração destes 10 sismogramas pseudo de-blended.

Finalmente, foram migrados os 100 sismogramas pseudo de-blended oriundos da aplicação deste esquema de pseudo decomposição de tiros nos 10 sismogramas gerados a partir de 10 experimentos com 10 tiros blended cada. As 100 imagens resultantes foram empilhadas de modo a gerarem as imagens finais apresentadas na figura 5 .

Nestas imagens pode-se observar e comentar que, a condição de imagem de correlação cruzada apresentou menor quantidade de ruído coerente na imagem final, se mostrando mais eficaz na geração de imagens a partir da aplicação da pseudo decomposição de tiros blended.

Ainda na figura 5 são apresentadas as imagens empilhadas obtidas da migração dos 10 sismogramas com 10 tiros blended cada. Fazendo uma comparação entre as imagens obtidas da migração de tiros pseudo 
de-blended com as obtidas da migração de tiros blended para o modelo com camadas plano-paralelas pode-se inferir que:

- Para a condição de imagem de correlação cruzada as imagens não apresentam divergências.

- Para a condição de imagem de tempo de excitação a imagem obtida da migração de tiros pseudo deblended apresentou maior relação sinal-ruído.
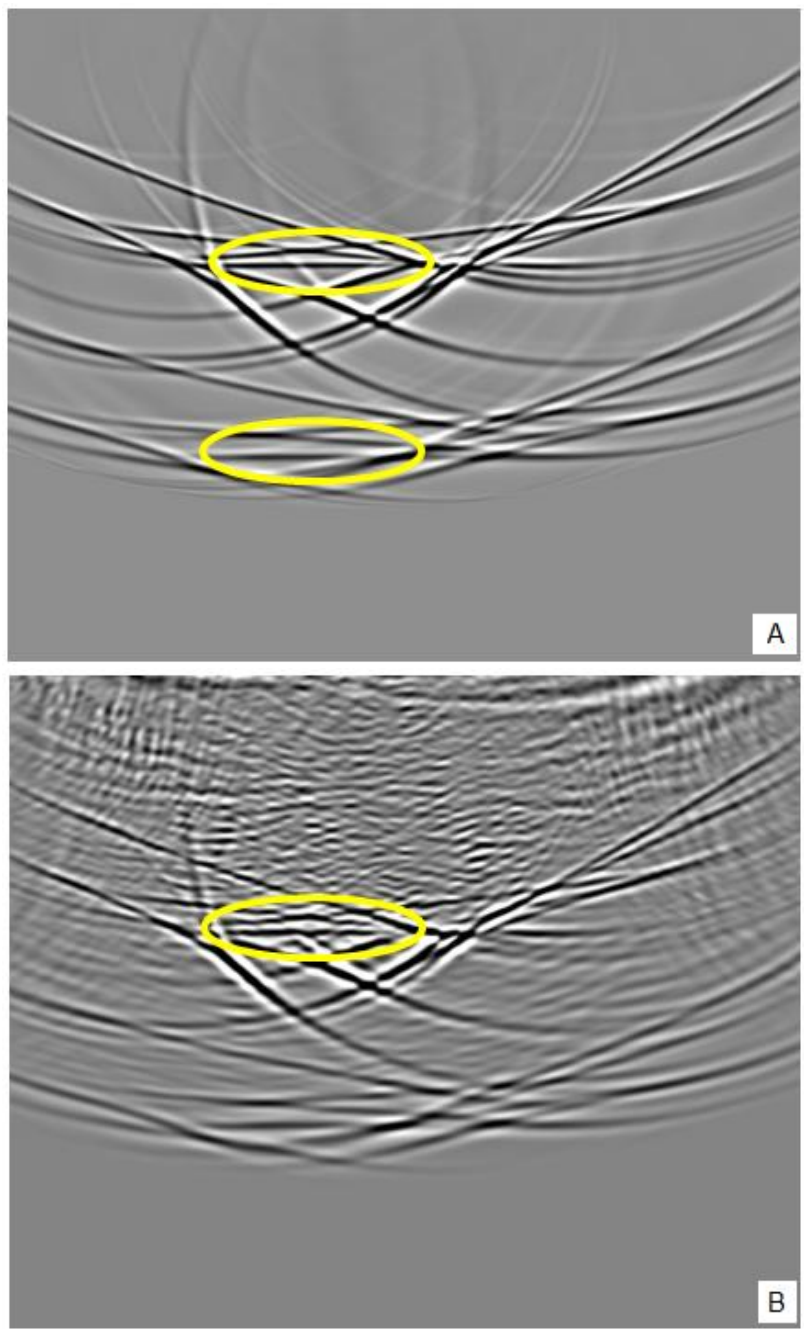

Figura 3 - Imagem obtida a partir da migração de 1 sismograma pseudo de-blended empregando condição de imagem de: $A$ ) tempo de excitação; B) correlacão cruzada.
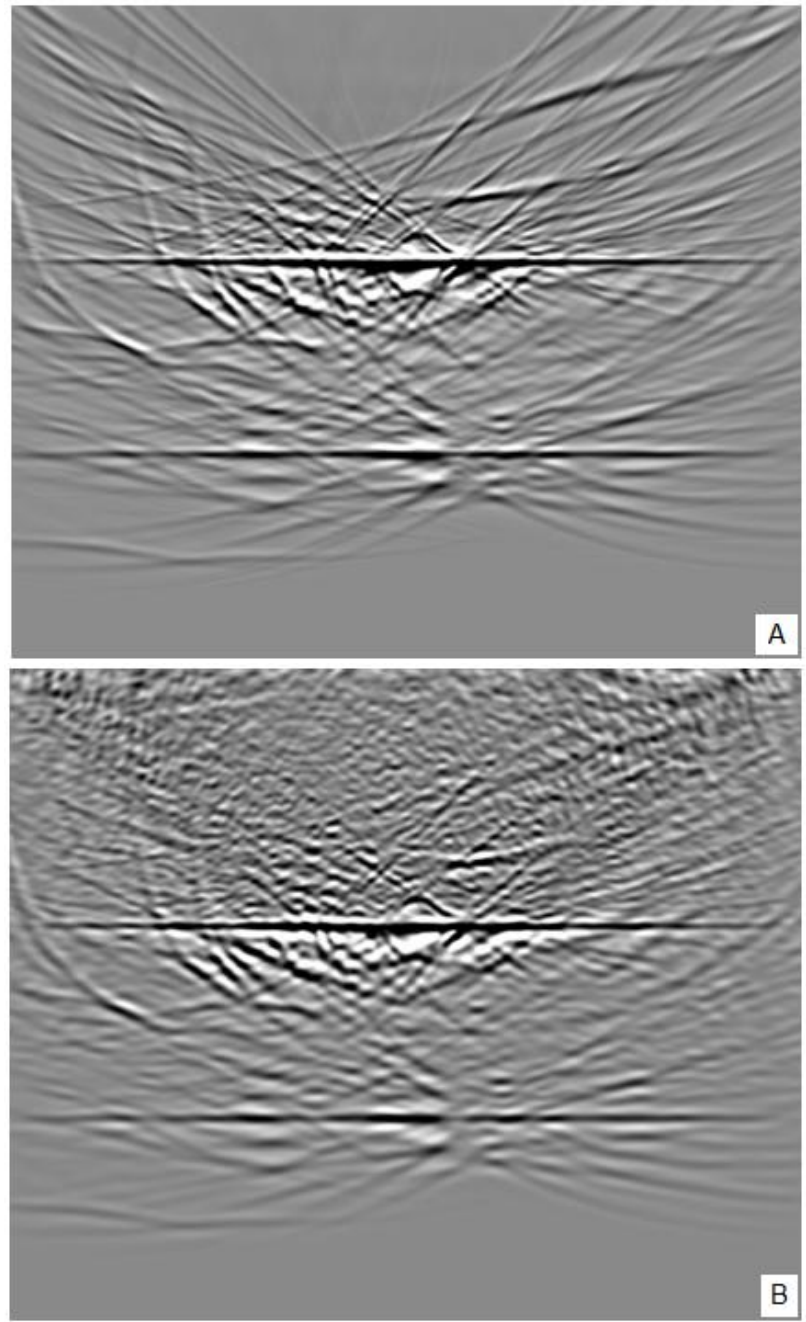

Figura 4 - Imagem obtida a partir do empilhamento de 10 imagens oriundas da migração de 10 sismogramas pseudo de-blended empregando condição de imagem de: $A$ ) tempo de excitação; B) correlação cruzada. 

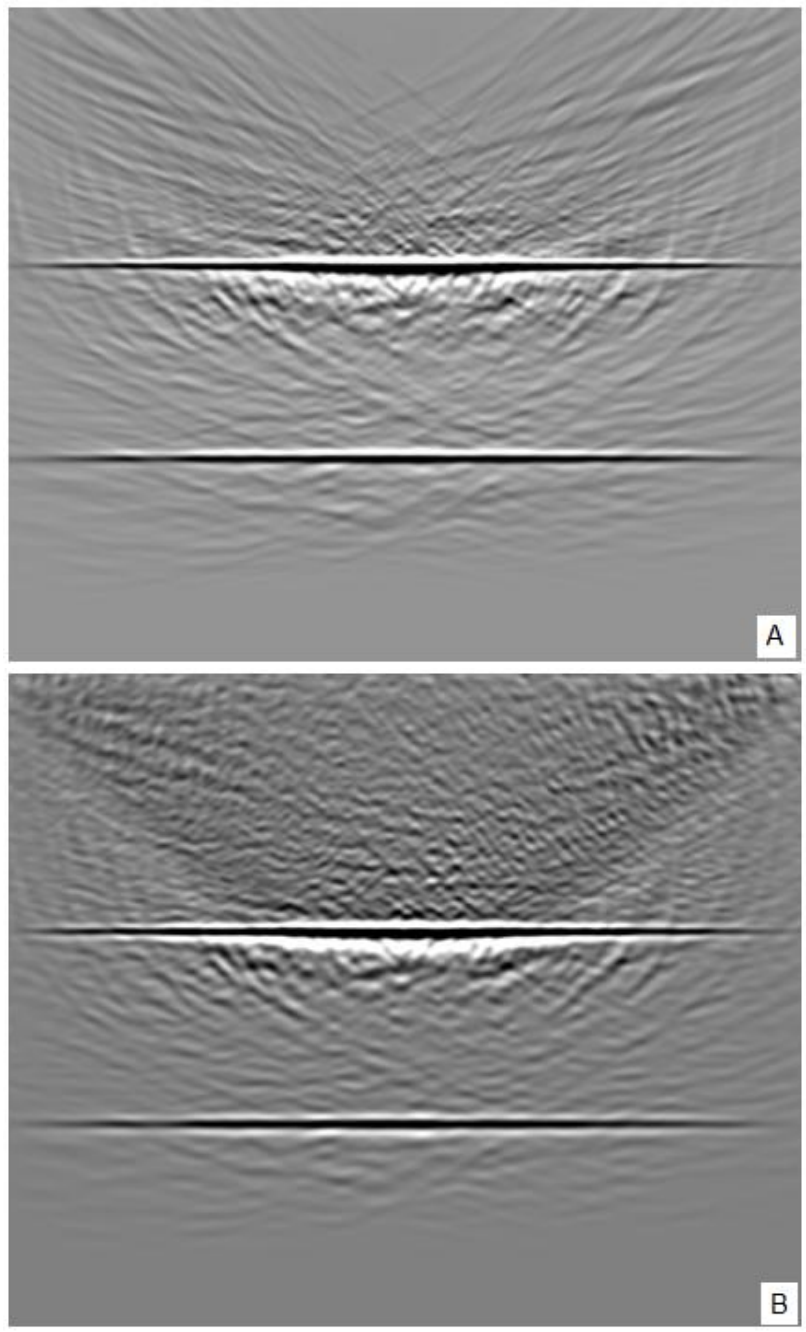
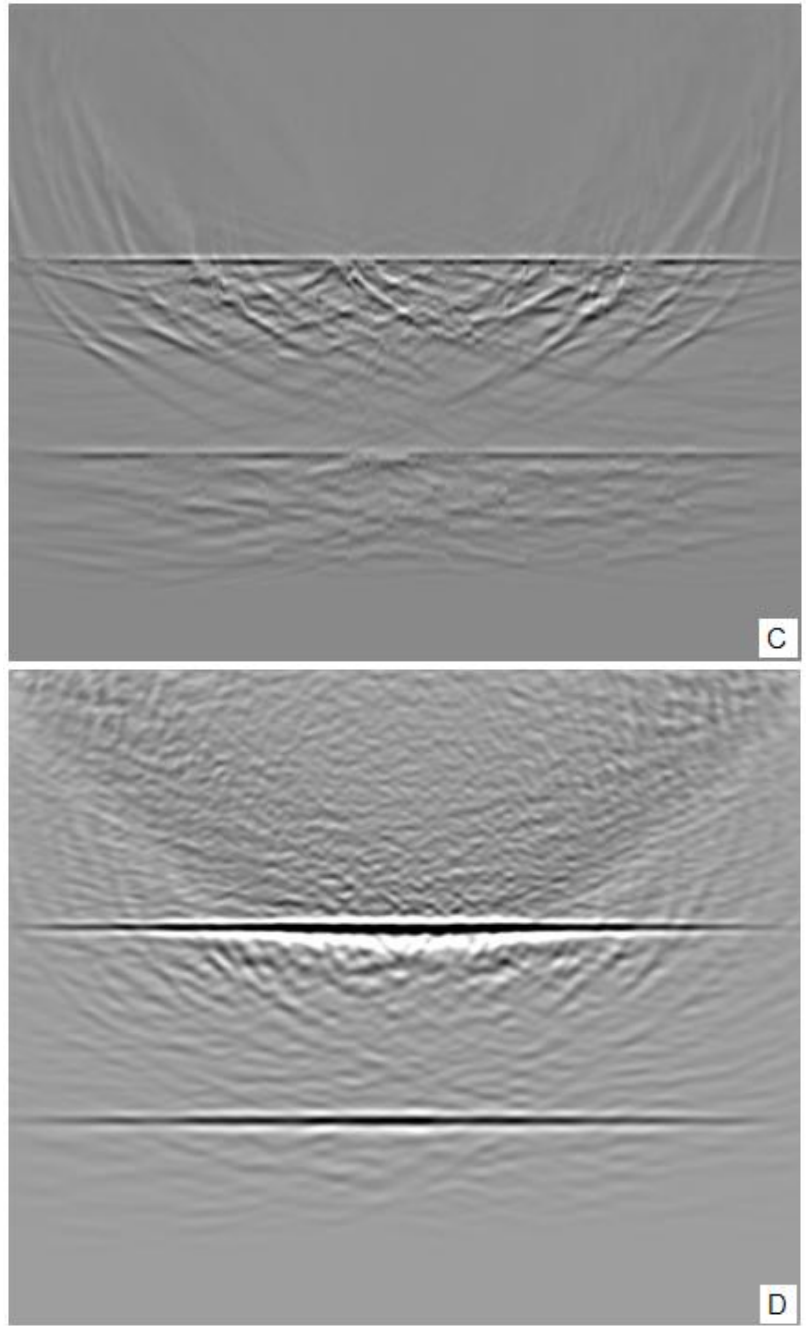

Figura 5 - (Esquerda) Imagens obtidas a partir do empilhamento de 100 imagens oriundas da migração dos 100 sismogramas pseudo de-blended empregando condição de imagem de: A) tempo de excitação; B) correlação cruzada. (Direita) Imagens obtidas a partir do empilhamento de 10 imagens oriundas da migração dos 10 sismogramas com 10 tiros blended cada empregando condição de imagem de: C) tempo de excitação; D) correlação cruzada.

\section{Discussão e Conclusões}

Como o próprio nome sugere e pode ser observado nas imagens apresentadas, 0 método de pseudo decomposição de tiros blended não consegue eliminar completamente a interferência entre as diversas fontes.

Fazendo uma comparação entre as imagens obtidas da migração de tiros pseudo de-blended com as obtidas da migração de tiros blended para o modelo com camadas plano-paralelas, observou-se que a condição de imagem de tempo de excitação apresentou imagem com maior relação sinal-ruído a partir da migração dos tiros pseudo de-blended. A condição de imagem de correlação cruzada não apresentou imagens com grandes divergências para os dois tipos de dados sísmicos.

Apesar da melhoria na qualidade da imagem para tiros pseudo de-blended empregando condição de imagem de tempo de excitação, o custo computacional na migração destes é muito maior que o requerido para migrar os tiros blended; neste último caso, para os tiros pseudo deblended foram feitas 100 migrações e para os tiros blended foram feitas apenas 10. Portanto, para um modelo geologicamente simples como o utilizado neste trabalho, a migração de tiros blended é suficiente para ambas as condições de imagem. 


\section{Agradecimentos}

Os autores da Universidade Federal do Rio de Janeiro agradecem à ANP pelo suporte financeiro e à Petrobras por autorizar esta publicação.

\section{Referências}

Berkhout, A.J., 2008. Changing the mindset in seismic data acquisition, The Leading Edge.

Claerbout, J.F., 1971. Toward a Unified Theory of Reflector Mapping, Geophysics, v.36, n.3, pp.467-481.

Clapp, R.G., 2009. Reverse Time Migration with Random Boundaries, Expanded Abstract, SEG, International and Annual Meeting, Houston.

Ikelle, L.T., 2007. Coding and Decoding: Seismic Data Modelling, Acquisition and Processing, Expanded Abstract, SEG, International and Annual Meeting, San Antonio.

Mahdad, A., Doulgeris, P., Blacquière, G. \& Campman, X., 2011. Separation of blended sources - Application to land seismic data. $73^{\text {rd }}$ EAGE Conference \& Exhibition incorporating SPE EUROPEC 2011, Vienna, Austria. 